\title{
Stability analysis for periodic solutions of fuzzy shunting inhibitory CNNs with delays
}

Ardak Kashkynbayev $^{1 *}$ (D) Jinde Cao ${ }^{2}$ and Zhaksybek Damiyev ${ }^{1}$

"Correspondence:

ardak.kashkynbayev@nu.edu.kz

'Department of Mathematics,

Nazarbayev University, Nur-Sultan,

Kazakhstan

Full list of author information is

available at the end of the article

\section{算 Springer}

\begin{abstract}
We consider fuzzy shunting inhibitory cellular neural networks (FSICNNs) with time-varying coefficients and constant delays. By virtue of continuation theorem of coincidence degree theory and Cauchy-Schwartz inequality, we prove the existence of periodic solutions for FSICNNs. Furthermore, by employing a suitable Lyapunov functional we establish sufficient criteria which ensure global exponential stability of the periodic solutions. Numerical simulations that support the theoretical discussions are depicted.
\end{abstract}

Keywords: Fuzzy shunting inhibitory cellular neural networks; Global exponential stability; Periodic solutions; Delay differential equations; Lyapunov functional

\section{Introduction and preliminaries}

In the past three decades, the cellular neural networks (CNNs) have gained a lot of popularity due to their local inter-connectivity and practical hardware implementation [1-8]. In terms of application viewpoint, the occurrence of time delays in CNNs is inevitable, which may lead to oscillations of CNNs as well as instability of the networks. For instance, when neural networks are implemented to the hardware there is time delay caused by the finite switching speed of the amplifier circuits [9]. Thus, it is of utmost importance to deal with delayed CNNs. On the other hand, one encounters to vagueness and lack of certainty in many real world application problems and the CNNs are not exception in this regard. The right instrument to deal with such problems is the so-called fuzzy logic theory which gives mathematical strength to handle uncertainties. Since the 1980s, this approach has been extensively applied to various engineering problems. However, it was only in 1996 Yang et al. introduced fuzzy cellular neural networks (FCNNs) in the series of papers [10, 11]. Along with pattern recognition and image processing, FCNNs play an essential role in cognitive science since human cognition involve many uncertainties. For this reason, the dynamics of FCNNs have been widely studied by many researchers. By means of direct Lyapunov method, exponential stability of FCNNs with different types of delays, diffusion and impulsive perturbations were established in many papers including [12-23]. Furthermore, the synchronization of FCNNs was considered in the studies [24-30]. On the other hand, from an application point of view, one is interested in a periodic or an almost periodic solution of FCNNs. Thus, the problems concerning the existence and stability of these solutions attracted many researchers and were investigated in the papers [31-34].

(c) The Author(s) 2019. This article is distributed under the terms of the Creative Commons Attribution 4.0 International License (http://creativecommons.org/licenses/by/4.0/), which permits unrestricted use, distribution, and reproduction in any medium, provided you give appropriate credit to the original author(s) and the source, provide a link to the Creative Commons license, and indicate if changes were made. 
A model of CNNs introduced by Bouzerdoum and Pinter [35] called shunting inhibitory cellular neural networks (SICNNs) have been applied in different disciplines including adaptive pattern recognition, psychophysics, image and signal processing, and cognitive science amongst others. For instance, the authors in the study [36] show that in addition to improving the color constancy of images, SICNNs fulfill contrast enhancement. Furthermore, in the study carried out by Arulampalam and Bouzerdoum [37], SICNNs were applied to medical diagnosis problems. In particular, these networks were used in medical classification problems such as Wisconsin breast cancer and the Pima Indians diabetes. In addition to these studies, there is a vast literature devoted to the asymptotic behavior of SICNNs [38-44]. However, there is no study that deals with the fuzziness of the SICNNs. Therefore, in the present article we propose to consider fuzzy shunting inhibitory cellular neural networks (FSICNNs). This is the main motivation of this paper. We make use of the continuation theorem of coincidence degree theory and Cauchy-Schwartz inequality to prove the existence of a periodic solution. Further, we prove that the periodic solution is globally exponentially stable. We verify our results by means of MATLAB software. It worth nothing to mention that the results can be validated by software like Maple, Python or Mathematica. The results of the present paper are highlighted below.

- Time-dependent coefficients and constant delays are taken into account in the stability analysis of FSICNNs.

- Sufficient conditions for the existence of periodic solutions to FSICNNs are derived by means of coincidence degree theory and inequality techniques like Cauchy-Schwartz inequality.

- Sufficient conditions for the for the global exponential stability of the periodic solutions to FSICNNs are derived.

- Numerical simulations which support the theoretical results are given.

In the present paper, we consider the description of the FSICNN in the following form:

$$
\begin{aligned}
\dot{x}_{i j}(t)= & -a_{i j}(t) x_{i j}(t)-\sum_{C_{k l} \in N_{r}(i, j)} C_{i j}^{k l}(t) f\left(x_{k l}(t)\right) x_{i j}(t)+L_{i j}(t) \\
& +\sum_{C_{k l} \in N_{r}(i, j)} B_{i j}^{k l}(t) U_{i j}(t)-\bigwedge_{C_{k l} \in N_{r}(i, j)} D_{i j}^{k l}(t) f\left(x_{k l}\left(t-\tau_{k l}\right)\right) x_{i j}(t) \\
& -\bigvee_{C_{k l} \in N_{r}(i, j)} E_{i j}^{k l}(t) f\left(x_{k l}\left(t-\tau_{k l}\right)\right) x_{i j}(t)+\bigwedge_{C_{k l} \in N_{r}(i, j)} T_{i j}^{k l}(t) U_{i j}(t) \\
& +\bigvee_{C_{k l} \in N_{r}(i, j)} H_{i j}^{k l}(t) U_{i j}(t),
\end{aligned}
$$

where $C_{i j}, i=1,2, \ldots, m, j=1,2, \ldots, n$, denote the cell at the $(i, j)$ position of the lattice, the $r$-neighborhood of $C_{i j}$ is $N_{r}(i, j)=\left\{C_{k l}: \max \{|k-i|,|l-j|\} \leq r, 1 \leq k \leq m, 1 \leq l \leq n\right\}$, $x_{i j}$ represent the activity of the cell $C_{i j}$ at time $t$; the positive functions $a_{i j}(t)$ are the passive decay rate of the cell activity; $U_{i j}(t)$ are the external input whereas $L_{i j}(t)$ are the external bias on the $(i, j)$ th cell; the nonnegative functions $C_{i j}^{k l}(t), D_{i j}^{k l}(t), E_{i j}^{k l}(t), T_{i j}^{k l}(t)$, and $H_{i j}^{k l}(t)$ are the connection or coupling strength of the postsynaptic activity, the fuzzy feedback MIN template, fuzzy feedback MAX template, fuzzy feed forward MIN template, and fuzzy feed forward MAX template of the cell $C_{k l}$ transmitted to the cell $C_{i j}$ at time $t$, respectively; $\bigwedge$ is the fuzzy AND operation whereas $\bigvee$ is the fuzzy OR operation; the functions $f\left(x_{k l}\right)$ represent the measures of activation to the output or firing rate of the 
cell $C_{k l}$; and $\tau_{k l}$ correspond to the transmission delay along the axon of the $(k, l)$ th cell from the $(i, j)$ th cell, $f(x)=\left(f_{11}(x), \ldots, f_{1 n}(x), \ldots, f_{m 1}(x), \ldots, f_{m n}(x)\right)^{T}, \Gamma_{1}=\{1,2, \ldots, m\}$ and $\Gamma_{2}=\{1,2, \ldots, n\}$.

We consider the network (1) subject to initial data

$$
x_{i j}(s)=\rho_{i j}(s), \quad s \in[-\tau, 0]
$$

where $\rho_{i j}(s)$ is the real-valued continuous function and $\tau=\max _{\substack{1 \leq k \leq m \\ 1 \leq l \leq n}}\left\{\tau_{k l}\right\}$.

The following notations will be used in the paper: $\|x\|=\sup _{-\tau \leq s \leq 0}|x(s)|$ is the norm in $\mathcal{C}([-\tau, 0], \mathbb{R})$, where $\mathcal{C}(A, B)$ is a set of continuous mappings from the space $A$ to the space $B, \bar{g}=\max _{t \in[0, \omega]}\{g(t)\}, \underline{g}=\min _{t \in[0, \omega]}\{g(t)\}$, and $\widetilde{g}=\frac{1}{\omega} \int_{0}^{\omega} g(t) d t$. Further, let $\mathbb{C}$ be a normed vector space then Dom $\mathbb{C}, \operatorname{Im} \mathbb{C}$ and $\operatorname{Ker} \mathbb{C}$ stand for the domain, image and kernel of the normed vector space $\mathbb{C}$, respectively.

Throughout the paper we will need the following assumptions.

(A1) The functions $a_{i j}(t), B_{i j}^{k l}(t), C_{i j}^{k l}(t), D_{i j}^{k l}(t), E_{i j}^{k l}(t), H_{i j}^{k l}(t), T_{i j}^{k l}(t), L_{i j}(t)$ and $U_{i j}(t)$ are continuous $\omega$-periodic functions for $i, k \in \Gamma_{1}, j, l \in \Gamma_{2}$.

(A2) The function $f(\cdot)$ is Lipschitz continuous on $\mathbb{R}$ with Lipschitz constants $L^{f}$ and satisfy $f(0)=0$, i.e., $|f(x)-f(y)| \leq L^{f}|x-y|$.

(A3) There exist constants $M$ and $m$ such that $m \leq|f(x)| \leq M$.

In order to show that there exists at least one periodic solution for the network (1), we utilize the continuation theorem of coincidence degree theory. Now, let us briefly summarize the technique introduced by Gaines and Mawhin in [45]. Consider normed vector spaces $\mathbb{X}$ and $\mathbb{Z}$. Suppose that $\mathcal{U}$ is a linear mapping such that $\operatorname{Dom} \mathcal{U} \subset \mathbb{X} \rightarrow \mathbb{Z}$ and $\mathcal{V}: \mathbb{X} \rightarrow \mathbb{Z}$ is a continuous mapping. If $\operatorname{dim} \operatorname{Ker} \mathcal{U}=\operatorname{Codim} \operatorname{Im} \mathcal{U}<\infty$ and $\operatorname{Im} \mathcal{U}$ is closed in $\mathbb{Z}$ then $\mathcal{U}$ is called a Fredholm mapping with index zero. For this case, there exist continuous projectors $\mathcal{A}: \mathbb{X} \rightarrow \mathbb{X}$ and $\mathcal{B}: \mathbb{Z} \rightarrow \mathbb{Z}$ which satisfy $\operatorname{Im} \mathcal{A}=\operatorname{Ker} \mathcal{U}$, and $\operatorname{Ker} \mathcal{B}=\operatorname{Im} \mathcal{U}=\operatorname{Im}(I-\mathcal{B})$. Then one can show that $\left.\mathcal{U}\right|_{\text {Dom } \mathcal{U} \cap \operatorname{Ker} \mathcal{A}}:(I-\mathcal{A}) \mathbb{X} \rightarrow \operatorname{Im} \mathcal{U}$ is invertible. For the convenience of the reader, denote the inverse mapping by $N_{\mathcal{A}}$. If $\mathcal{O}$ is any open bounded subset of $\mathbb{X}$, then the mapping $\mathcal{V}$ is called $L$-compact on $\overline{\mathcal{O}}$ if $\mathcal{B V}(\overline{\mathcal{O}})$ is bounded and $N_{\mathcal{A}}(I-\mathcal{B}) \mathcal{V}: \overline{\mathcal{O}} \rightarrow \mathbb{X}$ is compact. Further, one can show that there exists an isomorphism $\mathcal{F}: \operatorname{Im} \mathcal{B} \rightarrow \operatorname{Ker} \mathcal{U}$ since $\operatorname{Im} \mathcal{B}$ is isomorphic to $\operatorname{Ker} \mathcal{U}$.

Now, we are in the position to state the following lemma which will be useful in what follows.

Lemma 1.1 ([45]) Consider two normed spaces $\mathbb{X}$ and $\mathbb{Z}$ and let $\mathcal{U}:$ Dom $\mathcal{U} \subset \mathbb{X} \rightarrow \mathbb{Z}$ be a Fredholm operator with index zero. $\mathcal{O}$ is an open bounded subset of $\mathbb{X}$ and $\mathcal{V}: \mathbb{X} \rightarrow \mathbb{Z}$ is a continuous $L$-compact operator on $\overline{\mathcal{O}}$. Suppose that the following conditions hold true.

(i) $\mathcal{U} x \neq \mu \mathcal{V} x$ for each $\mu \in(0,1)$, and $x \in \operatorname{Dom} \mathcal{U} \cap \partial \mathcal{O}$;

(ii) $\mathcal{B} \mathcal{V} x \neq 0$ for each $x \in \operatorname{Ker} \mathcal{U} \cap \partial \mathcal{O}$;

(iii) $\operatorname{deg}\{\mathcal{F B V}, \operatorname{Ker} \mathcal{U} \cap \mathcal{O}, 0\} \neq 0$;

then the operator equation $\mathcal{U} x=\mathcal{V} x$ has at least one solution in $\operatorname{Dom} \mathcal{U} \cap \overline{\mathcal{O}}$.

In the sequel, we will need the following auxiliary lemma. The proof of the lemma is omitted since it can be easily shown by means of the result obtained by Yang et al. [46]. 
Lemma 1.2 Assume that $x, y \in \mathbb{R}$ are solutions of the network (1). Then the following inequalities hold true:

$$
\begin{aligned}
& \left|\bigwedge_{C_{k l} \in N_{r}(i, j)} D_{i j}^{k l}(t) f_{i j}(x)-\bigwedge_{C_{k l} \in N_{r}(i, j)} D_{i j}^{k l}(t) f_{i j}(y)\right| \\
& \leq \sum_{C_{k l} \in N_{r}(i, j)}\left|D_{i j}^{k l}(t)\right|\left|f_{i j}(x)-f_{i j}(y)\right|
\end{aligned}
$$

and

$$
\begin{aligned}
& \left|\bigvee_{C_{k l} \in N_{r}(i, j)} E_{i j}^{k l}(t) f_{i j}(x)-\bigvee_{C_{k l} \in N_{r}(i, j)} E_{i j}^{k l}(t) f_{i j}(y)\right| \\
& \leq \sum_{C_{k l} \in N_{r}(i, j)}\left|E_{i j}^{k l}(t)\right|\left|f_{i j}(x)-f_{i j}(y)\right| .
\end{aligned}
$$

\section{Existence and uniqueness of solutions}

In the present section, we prove the local existence and uniqueness of solutions to (1)-(2) by means of Banach fixed point theorem. To this end, set $a=\max _{i, j} a_{i j}$, $\delta=\max _{i, j}\left(\sum_{C_{k l} \in N_{r}(i, j)} \bar{C}_{i j}^{k l}+\sum_{C_{k l} \in N_{r}(i, j)} \bar{D}_{i j}^{k l}+\sum_{C_{k l} \in N_{r}(i, j)} \bar{E}_{i j}^{k l}\right)$ and $\gamma=\max _{i, j}\left(\bar{L}_{i j}+\right.$ $\left.\sum_{C_{k l} \in N_{r}(i, j)} \bar{B}_{i j}^{k l} \bar{U}_{i j}+\sum_{C_{k l} \in N_{r}(i, j)} \bar{T}_{i j}^{k l} \bar{U}_{i j}+\sum_{C_{k l} \in N_{r}(i, j)} \bar{H}_{i j} \bar{U}_{i j}\right)$.

One can show that a function $x(t)=\left\{x_{i j}(t)\right\}$ is a solution of the system (1)-(2) if and only if the following integral equation is satisfied:

$$
\begin{aligned}
x_{i j}(t)= & \rho_{i j}(0)+\int_{0}^{t}\left(-a_{i j}(s) x_{i j}(s)-\sum_{C_{k l} \in N_{r}(i, j)} C_{i j}^{k l}(s) f\left(x_{k l}(s)\right) x_{i j}(s)+L_{i j}(s)\right. \\
& +\sum_{C_{k l} \in N_{r}(i, j)} B_{i j}^{k l}(s) U_{i j}(s)-\bigwedge_{C_{k l} \in N_{r}(i, j)} D_{i j}^{k l}(s) f\left(x_{k l}\left(s-\tau_{k l}\right)\right) x_{i j}(s) \\
& -\bigvee_{C_{k l} \in N_{r}(i, j)} E_{i j}^{k l}(s) f\left(x_{k l}\left(s-\tau_{k l}\right)\right) x_{i j}(s) \\
& \left.+\bigwedge_{C_{k l} \in N_{r}(i, j)} T_{i j}^{k l}(s) U_{i j}(s)+\bigvee_{C_{k l} \in N_{r}(i, j)} H_{i j}^{k l}(s) U_{i j}(s)\right) d s, \quad t \geq 0 .
\end{aligned}
$$

Theorem 2.1 Assume that conditions (A1)-(A3) hold, then the system (1)-(2) has a unique solution.

Proof In order to prove the local existence and uniqueness we consider all functions $x_{i j}(t)$ on $[-\tau, \sigma]$ that satisfy $x_{i j}(t)=\rho_{i j}(t)$ on $[-\tau, 0]$ and $\left\|x_{i j}(t)-\rho_{i j}(t)\right\| \leq K$ on $(0, \sigma]$. Let us introduce a Banach space $\mathcal{C}_{0}$ to be all functions in $\mathcal{C}([-\tau, \sigma] ; \mathbb{R})$ such that $\left\|x_{i j}-\rho_{i j}\right\| \leq K$. Define an operator $\Pi$ on $\mathcal{C}_{0}$ as follows:

$$
(\Pi x)_{i j}(t)=\left\{\begin{array}{l}
\rho_{i j}(t), \quad t<0, \\
\rho_{i j}(0)+\int_{0}^{t}\left(-a_{i j}(s) x_{i j}(s)-\sum_{C_{k l} \in N_{r}(i, j)} C_{i j}^{k l}(s) f\left(x_{k l}(s)\right) x_{i j}(s)\right. \\
\quad+L_{i j}(s)+\sum_{C_{k l} \in N_{r}(i, j)} B_{i j}^{k l}(s) U_{i j}(s)-\bigwedge_{C_{k l} \in N_{r}(i, j)} D_{i j}^{k l}(s) f\left(x_{k l}\left(s-\tau_{k l}\right)\right) x_{i j}(s) \\
\quad-\bigvee_{C_{k l} \in N_{r}(i, j)} E_{i j}^{k l}(s) f\left(x_{k l}\left(s-\tau_{k l}\right)\right) x_{i j}(s) \\
\left.\quad+\bigwedge_{C_{k l} \in N_{r}(i, j)} T_{i j}^{k l}(s) U_{i j}(s)+\bigvee_{C_{k l} \in N_{r}(i, j)} H_{i j}^{k l}(s) U_{i j}(s)\right) d s, \quad t \geq 0 .
\end{array}\right.
$$


We begin by showing that $\Pi$ maps $\mathcal{C}_{0}$ into itself. Indeed,

$$
\begin{aligned}
\left|(\Pi x)_{i j}(t)-\rho_{i j}(0)\right| & \int_{0}^{t}\left(\left|a_{i j}(s)\right|\left|x_{i j}(s)\right|+\sum_{C_{k l} \in N_{r}(i, j)}\left|C_{i j}^{k l}(s)\right|\left|f\left(x_{k l}(s)\right)\right|\left|x_{i j}(s)\right|\right. \\
& +\left|L_{i j}(s)\right|+\sum_{C_{k l} \in N_{r}(i, j)}\left|B_{i j}^{k l}(s)\right|\left|U_{i j}(s)\right|+\bigwedge_{C_{k l} \in N_{r}(i, j)}\left|D_{i j}^{k l}(s)\right|\left|f\left(x_{k l}\left(s-\tau_{k l}\right)\right)\right|\left|x_{i j}(s)\right| \\
& +\bigvee_{C_{k l} \in N_{r}(i, j)}\left|E_{i j}^{k l}(s)\right|\left|f\left(x_{k l}\left(s-\tau_{k l}\right)\right)\right|\left|x_{i j}(s)\right| \\
& \left.+\bigwedge_{C_{k l} \in N_{r}(i, j)}\left|T_{i j}^{k l}(s)\right|\left|U_{i j}(s)\right|+\bigvee_{\mid C_{i j} \in N_{r}(i, j)}^{k l}(s)|| U_{i j}(s) \mid\right) d s \\
\leq & \int_{0}^{t}\left(\bar{a}_{i j} K+\sum_{C_{k l} \in N_{r}(i, j)} \bar{C}_{i j}^{k l} M K+\bar{L}_{i j}+\sum_{C_{k l} \in N_{r}(i, j)} \bar{B}_{i j}^{k l} \bar{U}_{i j}+\sum_{C_{k l} \in N_{r}(i, j)} \bar{D}_{i j}^{k l} M K\right. \\
& \left.+\sum_{C_{k l} \in N_{r}(i, j)} \bar{E}_{i j}^{k l} M K+\sum_{C_{k l} \in N_{r}(i, j)} \bar{T}_{i j}^{k l} \bar{U}_{i j}+\sum_{C_{k l} \in N_{r}(i, j)} \bar{H}_{i j} \bar{U}_{i j}\right) d s \\
\leq & \sigma(a K+M K \delta+\gamma) .
\end{aligned}
$$

We can choose $\sigma$ such that $\sigma(a K+M K \delta+\gamma) \leq K$. Thus, the last inequality yields $\Pi\left(\mathcal{C}_{0}\right) \subseteq$ $\mathcal{C}_{0}$.

On the other hand, for any $x(t), y(t) \in \mathcal{C}_{0}$ one can show that the following inequality hold's:

$$
\begin{aligned}
\left|(\Pi x)_{i j}(t)-(\Pi y)_{i j}(t)\right| & \\
\leq & \int_{0}^{t}\left(\left|a_{i j}(s)\right|\left|x_{i j}(s)-y_{i j}(s)\right|\right. \\
& +\sum_{C_{k l} \in N_{r}(i, j)}\left|C_{i j}^{k l}(s)\right|\left|f\left(x_{k l}(s)\right) x_{i j}(s)-f\left(y_{k l}(s)\right) y_{i j}(s)\right| \\
& +\bigwedge_{C_{k l} \in N_{r}(i, j)}\left|D_{i j}^{k l}(s)\right|\left|f\left(x_{k l}\left(s-\tau_{k l}\right)\right) x_{i j}(s)-f\left(y_{k l}\left(s-\tau_{k l}\right)\right) y_{i j}(s)\right| \\
& \left.+\bigvee_{C_{k l} \in N_{r}(i, j)}\left|E_{i j}^{k l}(s)\right|\left|f\left(x_{k l}\left(s-\tau_{k l}\right)\right) x_{i j}(s)-f\left(y_{k l}\left(s-\tau_{k l}\right)\right) y_{i j}(s)\right|\right) d s \\
\leq & \int_{0}^{t}\left(\left|\bar{a}_{i j}(s)\right|\left|x_{i j}(s)-y_{i j}(s)\right|+\sum_{C_{k l} \in N_{r}(i, j)} \bar{C}_{i j}^{k l}\left|f\left(x_{k l}(s)\right) x_{i j}(s)-f\left(y_{k l}(s)\right) x_{i j}(s)\right|\right. \\
& +\sum_{C_{k l} \in N_{r}(i, j)} \bar{C}_{i j}^{k l}\left|f\left(y_{k l}(s)\right) x_{i j}(s)-f\left(y_{k l}(s)\right) y_{i j}(s)\right| \\
& +\sum_{C_{k l} \in N_{r}(i, j)} \bar{D}_{i j}^{k l}\left|f\left(x_{k l}\left(s-\tau_{k l}\right)\right) x_{i j}(s)-f\left(y_{k l}\left(s-\tau_{k l}\right)\right) x_{i j}(s)\right| \\
& +\sum_{C_{k l} \in N_{r}(i, j)} \bar{D}_{i j}^{k l}\left|f\left(y_{k l}\left(s-\tau_{k l}\right)\right) x_{i j}(s)-f\left(y_{k l}\left(s-\tau_{k l}\right)\right) y_{i j}(s)\right|
\end{aligned}
$$




$$
\begin{aligned}
& +\sum_{C_{k l} \in N_{r}(i, j)} \bar{E}_{i j}^{k l}\left|f\left(x_{k l}\left(s-\tau_{k l}\right)\right) x_{i j}(s)-f\left(y_{k l}\left(s-\tau_{k l}\right)\right) x_{i j}(s)\right| \\
& \left.+\sum_{C_{k l} \in N_{r}(i, j)} \bar{E}_{i j}^{k l}\left|f\left(y_{k l}\left(s-\tau_{k l}\right)\right) x_{i j}(s)-f\left(y_{k l}\left(s-\tau_{k l}\right)\right) y_{i j}(s)\right|\right) d s \\
& \leq \int_{0}^{t}\left(\left|\bar{a}_{i j}(s)\right|\left|x_{i j}(s)-y_{i j}(s)\right|+\sum_{C_{k l} \in N_{r}(i, j)} \bar{C}_{i j}^{k l} M\left|x_{i j}(s)-y_{i j}(s)\right|\right. \\
& +\sum_{C_{k l} \in N_{r}(i, j)} \bar{C}_{i j}^{k l} K L^{f}\left|x_{i j}(s)-y_{i j}(s)\right|+\sum_{C_{k l} \in N_{r}(i, j)} \bar{D}_{i j}^{k l} M\left|x_{i j}(s)-y_{i j}(s)\right| \\
& +\sum_{C_{k l} \in N_{r}(i, j)} \bar{D}_{i j}^{k l} K L^{f}\left|x_{i j}\left(s-\tau_{k l}\right)-y_{i j}\left(s-\tau_{k l}\right)\right|+\sum_{C_{k l} \in N_{r}(i, j)} \bar{E}_{i j}^{k l} M\left|x_{i j}(s)-y_{i j}(s)\right| \\
& \left.+\sum_{C_{k l} \in N_{r}(i, j)} \bar{E}_{i j}^{k l} K L^{f}\left|x_{i j}\left(s-\tau_{k l}\right)-y_{i j}\left(s-\tau_{k l}\right)\right|\right) d s \\
& \leq \sigma\left(a+\delta\left(M+K L^{f}\right)\right) \sup _{t \geq-\tau}\left|x_{i j}(s)-y_{i j}(s)\right|=\sup _{t \geq-\tau}\left|x_{i j}(s)-y_{i j}(s)\right| .
\end{aligned}
$$

We can further choose $\sigma$ such that $\sigma\left(a+\delta\left(M+K L^{f}\right)\right)<1$. Thus, we have $\|\Pi x-\Pi y\| \leq$ $\sigma\left(a+\delta\left(M+K L^{f}\right)\right)\|x-y\|<\|x-y\|$ yielding the operator $\Pi$ is contractive. Consequently, the Banach fixed point theorem implies that the system (1)-(2) has a unique solution.

\section{Existence of periodic solutions}

In the present section, we prove the existence of periodic solutions of the network (1) by using the continuation theorem described in the previous section. That is, we utilize Lemma 1.1 to prove the existence of an $\omega$-periodic solution.

Theorem 3.1 Suppose that conditions (A1)-(A3) hold true, then the network (1)-(2) has at least one $\omega$-periodic solution.

Proof Set $\mathbb{X}=\mathbb{Z}=\left\{s(t) \in \mathcal{C}\left(\mathbb{R}, \mathbb{R}^{m n}\right), s(t+\omega)=s(t)\right\}$ equipped with the norm $\|s\|_{\omega}=$ $\max _{[0, \omega]} \sum_{i, j}\left|s_{i j}(t)\right|$. It can be easily seen that $\mathbb{X}$ and $\mathbb{Z}$ are Banach spaces with the norm $\|s\|_{\omega}$. Now, let us define $\mathcal{U} s=\frac{d s(t)}{d t}=\dot{s}$, where $\operatorname{Dom} \mathcal{U}=\mathcal{C}^{1}(\mathbb{X}, \mathbb{X})$ and further define projectors $\mathcal{A}$ as $\mathcal{A} x=\frac{1}{\omega} \int_{0}^{\omega} x(t) d t, x \in \mathbb{X}$, and $\mathcal{B}$ as $\mathcal{B} s=\frac{1}{\omega} \int_{0}^{\omega} s(t) d t, s \in \mathbb{Z}$, and the mapping $\mathcal{V}: \mathbb{X} \rightarrow \mathbb{X}$ as

$$
\begin{aligned}
\mathcal{V} x_{i j}(t)= & -a_{i j}(t) x_{i j}(t)-\sum_{C_{k l} \in N_{r}(i, j)} C_{i j}^{k l}(t) f_{i j}\left(x_{k l}(t)\right) x_{i j}(t) \\
& +L_{i j}(t)+\sum_{C_{k l} \in N_{r}(i, j)} B_{i j}^{k l}(t) U_{i j}(t) \\
& -\bigwedge_{C_{k l} \in N_{r}(i, j)} D_{i j}^{k l}(t) f_{i j}\left(x_{k l}\left(t-\tau_{k l}\right)\right) x_{i j}(t) \\
& -\bigvee_{C_{k l} \in N_{r}(i, j)} E_{i j}^{k l}(t) f_{i j}\left(x_{k l}\left(t-\tau_{k l}\right)\right) x_{i j}(t) \\
& +\bigwedge_{C_{k l} \in N_{r}(i, j)} T_{i j}^{k l}(t) U_{i j}(t)+\bigvee_{C_{k l} \in N_{r}(i, j)} H_{i j}^{k l}(t) U_{i j}(t) .
\end{aligned}
$$


One can verify that $\operatorname{Im} \mathcal{U}=\left\{s \mid s \in \mathbb{Z}: \int_{0}^{\omega} s(t) d t=0\right\}$ and $\operatorname{Ker} \mathcal{U}=\mathbb{R}^{m n}$. Thus, it follows that $\operatorname{Co} \operatorname{dim} \operatorname{Im} \mathcal{U}=\operatorname{dim} \operatorname{Ker} \mathcal{U}=m n$ and $\operatorname{Im} \mathcal{U}$ is closed in $\mathbb{Z}$. Therefore, all these imply that $\mathcal{U}$ is a Fredholm mapping with index zero. By the choice of projectors $\mathcal{A}$ and $\mathcal{B}$ we have $\operatorname{Im} \mathcal{A}=\operatorname{Ker} \mathcal{U}$ and $\operatorname{Ker} \mathcal{B}=\operatorname{Im}(I-\mathcal{B})=\operatorname{Im} \mathcal{U}$. Moreover, one can see that the inverse to $\mathcal{U}$ is defined as $N_{\mathcal{A}}: \operatorname{Im} \mathcal{U} \rightarrow \operatorname{Dom} \mathcal{U} \cap \operatorname{Ker} \mathcal{A}$ with $N_{\mathcal{A}}(s)=\int_{0}^{\omega} s(l) d l-\frac{1}{\omega} \int_{0}^{\omega} \int_{0}^{t} s(l) d l d t$. Clearly, both $\mathcal{B V}$ and $N_{\mathcal{A}}(I-\mathcal{B}) \mathcal{V}$ are continuous mappings. Finally, by virtue of Ascoli-Arzela lemma one can easily show that $\mathcal{B V}(\overline{\mathcal{O}})$ and $N_{\mathcal{A}}(I-\mathcal{B}) \mathcal{V}(\overline{\mathcal{O}})$ are relatively compact for an open and bounded $\mathcal{O}$ subset of $\mathbb{X}$. Thus, it follows that for any open and bounded set $\mathcal{O} \subset \mathbb{X}$ the mapping $\mathcal{V}$ is $L$-compact on $\overline{\mathcal{O}}$.

Now, it remains to find an open and bounded set $\mathcal{O}$ subset of $\mathbb{X}$ to apply Lemma 1.1. Thus, for each $\mu \in(0,1)$ the operator equation $\mathcal{U} x=\mu \mathcal{V} x$ yields

$$
\begin{aligned}
\dot{x}_{i j}(t)= & \mu\left[-a_{i j}(t) x_{i j}(t)-\sum_{C_{k l} \in N_{r}(i, j)} C_{i j}^{k l}(t) f_{i j}\left(x_{k l}(t)\right) x_{i j}(t)\right. \\
& +L_{i j}(t)+\sum_{C_{k l} \in N_{r}(i, j)} B_{i j}^{k l}(t) U_{i j}(t) \\
& -\bigwedge_{C_{k l} \in N_{r}(i, j)} D_{i j}^{k l}(t) f_{i j}\left(x_{k l}\left(t-\tau_{k l}\right)\right) x_{i j}(t) \\
& -\bigvee_{C_{k l} \in N_{r}(i, j)} E_{i j}^{k l}(t) f_{i j}\left(x_{k l}\left(t-\tau_{k l}\right)\right) x_{i j}(t) \\
& \left.+\bigwedge_{C_{k l} \in N_{r}(i, j)} T_{i j}^{k l}(t) U_{i j}(t)+\bigvee_{C_{k l} \in N_{r}(i, j)} H_{i j}^{k l}(t) U_{i j}(t)\right] .
\end{aligned}
$$

Next, after multiplying the system (4) with $x_{i j}(t)$ and integrating over the period, we derive

$$
\begin{aligned}
0= & \frac{1}{2}\left(x_{i j}^{2}(\omega)-x_{i j}^{2}(0)\right)=\int_{0}^{\omega} x_{i j}(t) \dot{x}_{i j}(t) d t \\
= & \mu\left\{-\int_{0}^{\omega} a_{i j}(t) x_{i j}^{2}(t) d t\right. \\
& -\int_{0}^{\omega} \sum_{C_{k l} \in N_{r}(i, j)} C_{i j}^{k l}(t) f_{i j}\left(x_{k l}(t)\right) x_{i j}^{2}(t) d t \\
& -\int_{0}^{\omega} \bigwedge_{C_{k l} \in N_{r}(i, j)} D_{i j}^{k l}(t) f_{i j}\left(x_{k l}\left(t-\tau_{k l}\right)\right) x_{i j}^{2}(t) d t \\
& -\int_{0}^{\omega} \bigvee_{C_{k l} \in N_{r}(i, j)} E_{i j}^{k l}(t) f_{i j}\left(x_{k l}\left(t-\tau_{k l}\right)\right) x_{i j}^{2}(t) d t \\
& +\int_{0}^{\omega} \sum_{C_{k l} \in N_{r}(i, j)} B_{i j}^{k l}(t) U_{i j}(t) x_{i j}(t) d t \\
& +\int_{0}^{\omega} \bigwedge_{C_{k l} \in N_{r}(i, j)} T_{i j}^{k l}(t) U_{i j}(t) x_{i j}(t) d t
\end{aligned}
$$




$$
\begin{aligned}
& +\int_{0}^{\omega} \bigvee_{C_{k l} \in N_{r}(i, j)} H_{i j}^{k l}(t) U_{i j}(t) x_{i j}(t) d t \\
& \left.+\int_{0}^{\omega} L_{i j}(t) x_{i j}(t) d t\right\}
\end{aligned}
$$

By means of the condition (A3), and Lemma 1.2 we get the following inequality:

$$
\begin{aligned}
\left(\underline{a}_{i j}\right. & \left.+\sum_{C_{k l} \in N_{r}(i, j)} \underline{C}_{i j}^{k l} m+\underline{D}_{i j}^{k l} m+\underline{E}_{i j}^{k l} m\right) \int_{0}^{\omega}\left|x_{i j}^{2}(t)\right| d t \\
\leq & \int_{0}^{\omega}\left|L_{i j}(t)\right|\left|x_{i j}(t)\right| d t \\
& +\int_{0}^{\omega} \sum_{C_{k l} \in N_{r}(i, j)}\left|B_{i j}^{k l}(t)\right|\left|U_{i j}(t)\right|\left|x_{i j}(t)\right| d t \\
& +\int_{0}^{\omega} \bigwedge_{C_{k l} \in N_{r}(i, j)}\left|T_{i j}^{k l}(t)\right|\left|U_{i j}(t)\right|\left|x_{i j}(t)\right| d t \\
& +\int_{0}^{\omega} \bigvee_{C_{k l} \in N_{r}(i, j)}\left|H_{i j}^{k l}(t)\right|\left|U_{i j}(t)\right|\left|x_{i j}(t)\right| d t \\
\leq & {\left[\bar{L}_{i j}+\sum_{C_{k l} \in N_{r}(i, j)} \bar{B}_{i j}^{k l} \bar{U}_{i j}+\left(\bar{T}_{i j}^{k l}+\bar{H}_{i j}^{k l}\right) \bar{U}_{i j}\right] \int_{0}^{\omega}\left|x_{i j}(t)\right| d t . }
\end{aligned}
$$

The Cauchy-Schwarz inequality yields $\int_{0}^{\omega}\left|x_{i j}(t)\right| d t \leq \sqrt{\omega}\left(\int_{0}^{\omega}\left|x_{i j}(t)\right|^{2} d t\right)^{\frac{1}{2}}$.

Then we get

$$
\left(\int_{0}^{\omega}\left|x_{i j}(t)\right|^{2} d t\right)^{\frac{1}{2}} \leq \frac{1}{G_{i j}}\left(\bar{L}_{i j}+\sum_{C_{k l} \in N_{r}(i, j)} \bar{B}_{i j}^{k l} \bar{U}_{i j}+\left(\bar{T}_{i j}^{k l}+\bar{H}_{i j}^{k l}\right) \bar{U}_{i j}\right) \sqrt{\omega}=Q_{i j},
$$

where $G_{i j}=\underline{a}_{i j}+\sum_{C_{k l} \in N_{r}(i, j)} \underline{C}_{i j}^{k l}(t) m+\underline{D}_{i j}^{k l}(t) m+\underline{E}_{i j}^{k l}(t) m$. Now, let $\beta \in[0, \omega]$ such that $\left|x_{i j}(\beta)\right|=\min _{t \in[0, \omega]}\left|x_{i j}(t)\right|$. Then, using (7) and the Cauchy-Schwarz inequality once again, we get the inequality

$$
\begin{aligned}
\left|x_{i j}(\beta)\right| \omega & =\left|x_{i j}(\beta)\right| \int_{0}^{\omega} d t \leq \int_{0}^{\omega}\left|x_{i j}(t)\right| d t \\
& \leq\left(\int_{0}^{\omega} 1^{2} d t\right)^{\frac{1}{2}}\left(\int_{0}^{\omega}\left|x_{i j}(t)\right|^{2} d t\right)^{\frac{1}{2}} \leq \sqrt{\omega} Q_{i j} .
\end{aligned}
$$

Hence,

$$
\left|x_{i j}(\beta)\right| \leq \frac{Q_{i j}}{\sqrt{\omega}} .
$$

Using (8) and $x_{i j}(t)=x_{i j}(\beta)+\int_{\beta}^{t} \dot{x}_{i j}(t) d t$, we have

$$
\left|x_{i j}(t)\right| \leq \frac{Q_{i j}}{\sqrt{\omega}}+\int_{\beta}^{\omega}\left|\dot{x}_{i j}(t)\right| d t .
$$


On the other hand, we have

$$
\begin{aligned}
& \int_{\beta}^{\omega}|\dot{x}(t)| d t \leq \int_{0}^{\omega}\left|a_{i j}(t)\right|\left|x_{i j}(t)\right| d t+\int_{0}^{\omega}\left|L_{i j}(t)\right| d t \\
& +\int_{0}^{\omega} \sum_{C_{k l} \in N_{r}(i, j)}\left|C_{i j}^{k l}(t)\right|\left|f_{i j}\left(x_{k l}(t)\right)\right|\left|x_{i j}(t)\right| d t \\
& +\int_{0}^{\omega} \sum_{C_{k l} \in N_{r}(i, j)}\left|B_{i j}^{k l}(t)\right|\left|U_{i j}(t)\right| d t \\
& +\int_{0}^{\omega} \bigwedge_{C_{k l} \in N_{r}(i, j)}\left|D_{i j}^{k l}(t)\right|\left|f_{i j}\left(x_{k l}\left(t-\tau_{k l}\right)\right)\right|\left|x_{i j}(t)\right| d t \\
& +\int_{0}^{\omega} \bigvee_{C_{k l} \in N_{r}(i, j)}\left|E_{i j}^{k l}(t)\right|\left|f_{i j}\left(x_{k l}\left(t-\tau_{k l}\right)\right)\right|\left|x_{i j}(t)\right| d t \\
& +\int_{0}^{\omega} \bigwedge_{C_{k l} \in N_{r}(i, j)}\left|T_{i j}^{k l}(t)\right|\left|U_{i j}(t)\right| d t \\
& +\int_{0}^{\omega} \bigvee_{C_{k l} \in N_{r}(i, j)}\left|H_{i j}^{k l}(t)\right|\left|U_{i j}(t)\right| d t \\
& \leq\left(\int_{0}^{\omega}\left|a_{i j}(t)\right|^{2} d t\right)^{\frac{1}{2}}\left(\int_{0}^{\omega}\left|x_{i j}(t)\right|^{2} d t\right)^{\frac{1}{2}}+\omega \bar{L}_{i j} \\
& +\sum_{C_{k l} \in N_{r}(i, j)} \bar{C}_{i j}^{k l}\left(\int_{0}^{\omega}\left|f_{i j}\left(x_{k l}(t)\right)\right|^{2} d t\right)^{\frac{1}{2}}\left(\int_{0}^{\omega}\left|x_{i j}(t)\right|^{2} d t\right)^{\frac{1}{2}} \\
& +\sum_{C_{k l} \in N_{r}(i, j)} \bar{B}_{i j}^{k l} \sqrt{\omega}\left(\int_{0}^{\omega}\left|U_{i j}(t)\right|^{2} d t\right)^{\frac{1}{2}} \\
& +\sum_{C_{k l} \in N_{r}(i, j)} \bar{D}_{i j}^{k l}\left(\int_{0}^{\omega}\left|f_{i j}\left(x_{k l}\left(t-\tau_{k l}\right)\right)\right|^{2} d t\right)^{\frac{1}{2}}\left(\int_{0}^{\omega}\left|x_{i j}(t)\right|^{2} d t\right)^{\frac{1}{2}} \\
& +\sum_{C_{k l} \in N_{r}(i, j)} \bar{E}_{i j}^{k l}\left(\int_{0}^{\omega}\left|f_{i j}\left(x_{k l}\left(t-\tau_{k l}\right)\right)\right|^{2} d t\right)^{\frac{1}{2}}\left(\int_{0}^{\omega}\left|x_{i j}(t)\right|^{2} d t\right)^{\frac{1}{2}} \\
& +\omega \sum_{C_{k l} \in N_{r}(i, j)}\left(\bar{T}_{i j}^{k l}+\bar{H}_{i j}^{k l}\right) \bar{U}_{i j} \\
& \leq \sqrt{\omega} \cdot \bar{a}_{i j} Q_{i j} \\
& +\omega \bar{L}_{i j}+\sqrt{\omega} \sum_{C_{k l} \in N_{r}(i, j)} \bar{C}_{i j}^{k l} M Q_{i j}+\sqrt{\omega} \sum_{C_{k l} \in N_{r}(i, j)} \bar{B}_{i j}^{k l} \bar{U}_{i j} \\
& +\sqrt{\omega}\left(\bar{D}_{i j}^{k l}+\bar{E}_{i j}^{k l}\right) M Q_{i j}+\omega \sum_{C_{k l} \in N_{r}(i, j)}\left(\bar{T}_{i j}^{k l}+\bar{H}_{i j}^{k l}\right) \bar{U}_{i j} \\
& =V_{i j} \text {. }
\end{aligned}
$$

Thus, it follows from (9) that

$$
\left|x_{i j}(t)\right| \leq \frac{Q_{i j}}{\sqrt{\omega}}+V_{i j}=S_{i j} .
$$


Denote $P=\sum_{(i, j)} S_{i j}+Q$, where $Q>0$ is chosen so that the following inequality is satisfied: $\min _{\substack{1 \leq i \leq m \\ 1 \leq j \leq n}}\left(\widetilde{a}_{i j}+\widetilde{C}_{i j}^{k l} m+\widetilde{D}_{i j}^{k l} m+\widetilde{E}_{i j}^{k l} m\right) P>m n \max _{\substack{1 \leq i \leq m \\ 1 \leq j \leq n}}\left(\left|\widetilde{L}_{i j}\right|+\sum_{C_{k l} \in N_{r}(i, j)}\left|\bar{B}_{i j}^{k l}\right|\left|\bar{U}_{i j}\right|+\right.$ $\left.\sum_{C_{k l} \in N_{r}(i, j)}\left|\bar{T}_{i j}^{k l}\right|\left|\bar{U}_{i j}\right|+\sum_{C_{k l} \in N_{r}(i, j)}\left|\bar{H}_{i j}^{k l}\right|\left|\bar{U}_{i j}\right|\right)$.

If we choose $\mathcal{O}=\left\{x \in \mathbb{R}^{m n} \mid\|x\|_{\omega}<P\right\}$, then $\mathcal{O} \subset \mathbb{X}$ is an open and bounded set. Further, one can show that $\mathcal{O}$ fulfills the condition (i) of Lemma 1.1.

Next, let us verify the condition (ii) of Lemma 1.1. That is, we need to show that $\mathcal{B V} x \neq 0$. If $x \in \operatorname{Ker} \mathcal{U} \cap \partial \mathcal{O}$ then $x \in \mathbb{R}^{m n}$ is a constant vector and satisfies $\|x\|_{\omega}=\left|x_{11}\right|+\cdots+\left|x_{1 n}\right|+$ $\cdots+\left|x_{m 1}\right|+\cdots+\left|x_{m n}\right|=P$. Therefore, we have

$$
\begin{aligned}
\mathcal{B V} x= & -\tilde{a}_{i j} x_{i j}-\sum_{C_{k l} \in N_{r}(i, j)} \widetilde{C}_{i j}^{k l} f_{i j}\left(x_{k l}\right) x_{i j}+\widetilde{L}_{i j} \\
& +\sum_{C_{k l} \in N_{r}(i, j)} \widetilde{B_{i j}^{k l} U_{i j}}-\bigwedge_{C_{k l} \in N_{r}(i, j)} \widetilde{D}_{i j}^{k l} f_{i j}\left(x_{k l}\right) x_{i j} \\
& -\bigvee_{C_{k l} \in N_{r}(i, j)} \widetilde{E_{i j}^{k l}} f_{i j}\left(x_{k l}\right) x_{i j}+\bigwedge_{C_{k l} \in N_{r}(i, j)} \widetilde{T_{i j}^{k l} U_{i j}} \\
& +\bigvee_{C_{k l} \in N_{r}(i, j)} \widetilde{H_{i j}^{k l} U_{i j}}
\end{aligned}
$$

The last expression yields

$$
\begin{aligned}
& \|\mathcal{B} \mathcal{V} x\|_{\omega}=\sum_{(i, j)} \mid \tilde{a}_{i j} x_{i j}+\sum_{C_{k l} \in N_{r}(i, j)} \widetilde{C}_{i j}^{k l} f_{i j}\left(x_{k l}\right) x_{i j}-\widetilde{L}_{i j} \\
& -\sum_{C_{k l} \in N_{r}(i, j)} \widetilde{B_{i j}^{k l} U_{i j}}+\bigwedge_{C_{k l} \in N_{r}(i, j)} \widetilde{D}_{i j}^{k l} f_{i j}\left(x_{k l}\right) x_{i j} \\
& +\bigvee_{C_{k l} \in N_{r}(i, j)} \widetilde{E}_{i j}^{k l} f_{i j}\left(x_{k l}\right) x_{i j}-\bigwedge_{C_{k l} \in N_{r}(i, j)} \widetilde{T_{i j}^{k l} U_{i j}} \\
& -\bigvee_{C_{k l} \in N_{r}(i, j)} \widetilde{H_{i j}^{k l} U_{i j}} \\
& \geq \sum_{(i, j)} \mid \bar{a}_{i j} x_{i j}+\sum_{C_{k l} \in N_{r}(i, j)} \widetilde{C}_{i j}^{k l} f_{i j}\left(x_{k l}\right) x_{i j} \\
& +\bigwedge_{C_{k l} \in N_{r}(i, j)} \widetilde{D}_{i j}^{k l} f_{i j}\left(x_{k l}\right) x_{i j}+\bigvee_{C_{k l} \in N_{r}(i, j)} \widetilde{E}_{i j}^{k l} f_{i j}\left(x_{k l}\right) x_{i j} \mid \\
& -\sum_{(i, j)} \mid \widetilde{L}_{i j}+\sum_{C_{k l} \in N_{r}(i, j)} \widetilde{B_{i j}^{k l} U_{i j}}+\bigwedge_{C_{k l} \in N_{r}(i, j)} \widetilde{T_{i j}^{k l} U_{i j}} \\
& +\bigvee_{C_{k l} \in N_{r}(i, j)} \widetilde{H_{i j}^{k l} U_{i j}} \mid \\
& \geq \min _{\substack{1 \leq i \leq m \\
1 \leq j \leq n}}\left(\widetilde{a}_{i j}+\widetilde{C}_{i j}^{k l} m+\widetilde{D}_{i j}^{k l} m+\widetilde{E}_{i j}^{k l} m\right) \sum_{(i, j)}\left|x_{i j}\right| \\
& -\max _{\substack{1 \leq i \leq m \\
1 \leq j \leq n}} m n\left(\left|\widetilde{L}_{i j}\right|+\sum_{C_{k l} \in N_{r}(i, j)}\left|\bar{B}_{i j}^{k l}\right|\left|\bar{U}_{i j}\right|\right.
\end{aligned}
$$




$$
\begin{aligned}
& \left.+\sum_{C_{k l} \in N_{r}(i, j)}\left|\bar{T}_{i j}^{k l}\right|\left|\bar{U}_{i j}\right|+\sum_{C_{k l} \in N_{r}(i, j)}\left|\bar{H}_{i j}^{k l}\right|\left|\bar{U}_{i j}\right|\right) \\
& >0,
\end{aligned}
$$

by the choice of $P$. Thus, for any $x \in \operatorname{Ker} \mathcal{U} \cap \partial \mathcal{O}$, we have $\mathcal{B V} x \neq 0$ which validates the requirement (ii) of Lemma 1.1.

Finally, consider homotopic $\Phi\left(x_{11}, \ldots, x_{1 n}, \ldots, x_{m 1} \ldots, x_{m n}, \lambda\right)=\lambda \mathcal{B} \mathcal{V} x+(1-\lambda) \mathcal{G} x$, where $\lambda \in[0,1]$ and $\mathcal{G} x=\left(-\widetilde{a}_{11} x_{11}, \ldots,-\widetilde{a}_{1 n} x_{1 n}, \ldots,-\widetilde{a}_{m 1} x_{m 1} \ldots,-\widetilde{a}_{m n} x_{m n}\right)^{T}$. Setting $\mathcal{F}=I$ one can easily show that

$$
\begin{gathered}
\operatorname{deg}\left\{\mathcal{F B V}\left(x_{11}, \ldots, x_{1 n}, \ldots, x_{m 1} \ldots, x_{m n}\right)^{T}, \operatorname{Ker} \mathcal{U} \cap \mathcal{O}, 0\right\} \\
=\operatorname{sgn}\left\{(-1)^{m n} \widetilde{a}_{11} \ldots \tilde{a}_{1 n} \ldots \widetilde{a}_{m 1} \ldots \tilde{a}_{m n}\right\} \neq 0 .
\end{gathered}
$$

Thus, the final requirement (iii) of Lemma 1.1 is also verified. As a result, $\mathcal{O}$ fulfills all of the necessities of Lemma 1.1. Consequently, the operator equation $\mathcal{U} x=\mathcal{V} x$ has at least one solution in Dom $\mathcal{U} \cap \overline{\mathcal{O}}$ which in turn implies that the network (1) has $\omega$-periodic solution. This is the end of the proof.

\section{Stability}

This part of the paper is devoted to the uniqueness and stability analysis of the periodic solutions. That is, we prove that there is a unique $\omega$-periodic solution of the network (1) and further prove that it is globally exponentially stable under a suitable condition. We assume the following condition holds.

$$
\text { (A4) } \begin{aligned}
\underline{a}_{i j}-M\left(\sum_{C_{k l} \in N_{r}(i, j)} \bar{C}_{i j}^{k l}+\sum_{C_{k l} \in N_{r}(i, j)} \bar{D}_{i j}^{k l}+\sum_{C_{k l} \in N_{r}(i, j)} \bar{E}_{i j}^{k l}\right)-S_{i j} L_{i j}\left(\sum_{C_{k l} \in N_{r}(i, j)} \bar{C}_{i j}^{k l}+\right. \\
\left.\sum_{C_{k l} \in N_{r}(i, j)} \bar{D}_{i j}^{k l}+\sum_{C_{k l} \in N_{r}(i, j)} \bar{E}_{i j}^{k l}\right)>0 .
\end{aligned}
$$

Theorem 4.1 If the conditions (A1)-(A4) are fulfilled, then the network (1) has a unique globally exponentially stable $\omega$-periodic solution.

Proof In the previous section, we have shown that the network (1) has an $\omega$-periodic solution. Let us consider arbitrary two solutions of the network $(1) x(t)=\left(x_{11}(t), \ldots, x_{1 n}(t), \ldots\right.$, $\left.x_{m 1}(t) \ldots, x_{m n}(t)\right)$ and $y(t)=\left(y_{11}(t), \ldots, y_{1 n}(t), \ldots, y_{m 1}(t) \ldots, y_{m n}(t)\right)$ with the initial conditions $x(s)=\rho(s), s \in[-\tau, 0]$, and $y(s)=\xi(s), s \in[-\tau, 0]$, respectively. It is straightforward that the uniqueness of the solution for the network (1) is followed by global exponential stability. Hence, we only need to show that $x(t)$ is globally exponentially stable. By the condition (A4), one can find $\alpha>0$ so that the following inequality holds true: $\alpha-\underline{a}_{i j}+M\left(\sum_{C_{k l} \in N_{r}(i, j)} \bar{C}_{i j}^{k l}+\sum_{C_{k l} \in N_{r}(i, j)} \bar{D}_{i j}^{k l}+\sum_{C_{k l} \in N_{r}(i, j)} \bar{E}_{i j}^{k l}\right)+S_{i j} L^{f}\left(\sum_{C_{k l} \in N_{r}(i, j)} \bar{C}_{i j}^{k l}+\right.$ $\left.e^{\alpha \tau} \sum_{C_{k l} \in N_{r}(i, j)} \bar{D}_{i j}^{k l}+e^{\alpha \tau} \sum_{C_{k l} \in N_{r}(i, j)} \bar{E}_{i j}^{k l}\right)<0$.

We design a Lyapunov functional $W(t)=W_{1}(t)+W_{2}(t)$, given by

$$
\begin{aligned}
& W_{1}(t)=\sum_{(i, j)}\left|x_{i j}(t)-y_{i j}(t)\right| e^{\alpha t} \text {, and } \\
& W_{2}(t)= \sum_{(i, j)} S_{i j}\left(\sum_{C_{k l} \in N_{r}(i, j)} \bar{D}_{i j}^{k l}+\sum_{C_{k l} \in N_{r}(i, j)} \bar{E}_{i j}^{k l}\right) \\
& \times \int_{t-\tau_{k l}}^{t}\left|f_{i j}\left(x_{k l}(s)\right)-f_{i j}\left(y_{k l}(s)\right)\right| e^{\alpha\left(s+\tau_{k l}\right)} d s .
\end{aligned}
$$


Let us calculate upper right Dini derivatives of $W_{1}(t)$ and $W_{2}(t)$ along the trajectories of the network (1), respectively:

$$
\begin{aligned}
& \frac{d^{+} W_{1}(t)}{d t} \leq \sum_{(i, j)}\left[\left(-a_{i j}(t)\left|x_{i j}(t)-y_{i j}(t)\right|\right.\right. \\
& +\sum_{C_{k l} \in N_{r}(i, j)} C_{i j}^{k l}(t)\left|f_{i j}\left(x_{k l}(t)\right) x_{i j}(t)-f_{i j}\left(y_{k l}(t)\right) y_{i j}(t)\right| \\
& +\bigwedge_{C_{k l} \in N_{r}(i, j)} D_{i j}^{k l}(t) \mid f_{i j}\left(x_{k l}\left(t-\tau_{k l}\right)\right) x_{i j}(t) \\
& -f_{i j}\left(y_{k l}\left(t-\tau_{k l}\right)\right) y_{i j}(t) \mid \\
& +\bigvee_{C_{k l} \in N_{r}(i, j)} E_{i j}^{k l}(t) \mid f_{i j}\left(x_{k l}\left(t-\tau_{k l}\right)\right) x_{i j}(t) \\
& \left.\left.-f_{i j}\left(y_{k l}\left(t-\tau_{k l}\right)\right) y_{i j}(t) \mid\right) e^{\alpha t}+\alpha\left|x_{i j}(t)-y_{i j}(t)\right| e^{\alpha t}\right] \\
& \leq e^{\alpha t} \sum_{(i, j)}\left[\left(\alpha-\underline{a}_{i j}\right)\left|x_{i j}(t)-y_{i j}(t)\right|\right. \\
& +\sum_{C_{k l} \in N_{r}(i, j)} \bar{C}_{i j}^{k l}\left(\left|f_{i j}\left(x_{k l}(t)\right)\right|\left|x_{i j}(t)-y_{i j}(t)\right|\right. \\
& \left.+\left|f_{i j}\left(x_{k l}(t)\right)-f_{i j}\left(y_{k l}(t)\right)\right|\left|y_{k l}(t)\right|\right) \\
& +\left(\sum_{C_{k l} \in N_{r}(i, j)} \bar{D}_{i j}^{k l}+\sum_{C_{k l} \in N_{r}(i, j)} \bar{E}_{i j}^{k l}\right) \\
& \times\left(\left|f_{i j}\left(x_{k l}\left(t-\tau_{k l}\right)\right)\right|\left|x_{i j}(t)-y_{i j}(t)\right|\right. \\
& \left.\left.+\left|f_{i j}\left(x_{k l}\left(t-\tau_{k l}\right)\right)-f_{i j}\left(y_{k l}\left(t-\tau_{k l}\right)\right)\right|\left|y_{k l}(t)\right|\right)\right] \\
& \leq e^{\alpha t} \sum_{(i, j)}\left[\left(\alpha-\underline{a}_{i j}\right)\left|x_{i j}(t)-y_{i j}(t)\right|\right. \\
& +\sum_{C_{k l} \in N_{r}(i, j)} \bar{C}_{i j}^{k l}\left(M\left|x_{i j}(t)-y_{i j}(t)\right|\right. \\
& \left.+\left|f_{i j}\left(x_{k l}(t)\right)-f_{i j}\left(y_{k l}(t)\right)\right| S_{i j}\right) \\
& +\left(\sum_{C_{k l} \in N_{r}(i, j)} \bar{D}_{i j}^{k l}+\sum_{C_{k l} \in N_{r}(i, j)} \bar{E}_{i j}^{k l}\right) \\
& \times\left(M\left|x_{i j}(t)-y_{i j}(t)\right|\right. \\
& \left.\left.+\left|f_{i j}\left(x_{k l}\left(t-\tau_{k l}\right)\right)-f_{i j}\left(y_{k l}\left(t-\tau_{k l}\right)\right)\right| S_{i j}\right)\right] \\
& \leq e^{\alpha t} \sum_{(i, j)}\left[\left(\alpha-\underline{a}_{i j}+M\left(\sum_{C_{k l} \in N_{r}(i, j)} \bar{C}_{i j}^{k l}\right.\right.\right. \\
& \left.\left.+\sum_{C_{k l} \in N_{r}(i, j)} \bar{D}_{i j}^{k l}+\sum_{C_{k l} \in N_{r}(i, j)} \bar{E}_{i j}^{k l}\right)\right)\left|x_{i j}(t)-y_{i j}(t)\right|
\end{aligned}
$$




$$
\begin{aligned}
& +S_{i j}\left(L^{f} \sum_{C_{k l} \in N_{r}(i, j)} \bar{C}_{i j}^{k l}\left|x_{k l}(t)-y_{k l}(t)\right|+\left(\sum_{C_{k l} \in N_{r}(i, j)} \bar{D}_{i j}^{k l}\right.\right. \\
& \left.\left.\left.+\sum_{C_{k l} \in N_{r}(i, j)} \bar{E}_{i j}^{k l}\right)\left|f_{i j}\left(x_{k l}\left(t-\tau_{k l}\right)\right)-f_{i j}\left(y_{k l}\left(t-\tau_{k l}\right)\right)\right|\right)\right], \\
\frac{d^{+} W_{2}(t)}{d t}= & e^{\alpha t} \sum_{(i, j)} S_{i j}\left(\sum_{C_{k l} \in N_{r}(i, j)} \bar{D}_{i j}^{k l}+\sum_{C_{k l} \in N_{r}(i, j)} \bar{E}_{i j}^{k l}\right) \\
& \times\left[\left|f_{i j}\left(x_{k l}(t)\right)-f_{i j}\left(y_{k l}(t)\right)\right| e^{\alpha \tau_{k l}}\right. \\
& \left.-\left|f_{i j}\left(x_{k l}\left(t-\tau_{k l}\right)\right)-f_{i j}\left(y_{k l}\left(t-\tau_{k l}\right)\right)\right|\right] .
\end{aligned}
$$

Thus, by means of the above inequalities one can easily obtain

$$
\begin{aligned}
\frac{d^{+} W(t)}{d t}= & \frac{d^{+} W_{1}(t)}{d t}+\frac{d^{+} W_{2}(t)}{d t} \\
\leq & e^{\alpha t} \sum_{(i, j)}\left[\left(\alpha-\underline{a}_{i j}\right.\right. \\
& \left.+M\left(\sum_{C_{k l} \in N_{r}(i, j)} \bar{C}_{i j}^{k l}+\sum_{C_{k l} \in N_{r}(i, j)} \bar{D}_{i j}^{k l}+\sum_{C_{k l} \in N_{r}(i, j)} \bar{E}_{i j}^{k l}\right)\right) \\
& \times\left|x_{i j}(t)-y_{i j}(t)\right|+S_{i j}\left(L^{f} \sum_{C_{k l} \in N_{r}(i, j)} \bar{C}_{i j}^{k l}\left|x_{i j}(t)-y_{i j}(t)\right|\right. \\
& +\left(\sum_{C_{k l} \in N_{r}(i, j)} \bar{D}_{i j}^{k l}+\sum_{C_{k l} \in N_{r}(i, j)} \bar{E}_{i j}^{k l}\right) \\
& \left.\left.\times\left|f_{i j}\left(x_{k l}(t)\right)-f_{i j}\left(y_{k l}(t)\right)\right| e^{\alpha \tau_{k l}}\right)\right] \\
& \leq e^{\alpha t} \sum_{(i, j)}\left[\alpha-\underline{a}_{i j}+M\left(\sum_{C_{k l} \in N_{r}(i, j)} \bar{C}_{i j}^{k l}+\sum_{C_{k l} \in N_{r}(i, j)} \bar{D}_{i j}^{k l}\right.\right. \\
& \left.+\sum_{C_{k l} \in N_{r}(i, j)} \bar{E}_{i j}^{k l}\right)+S_{i j} L_{i j}\left(\sum_{C_{k l} \in N_{r}(i, j)} \bar{C}_{i j}^{k l}\right. \\
& \left.\left.+e^{\alpha \tau} \sum_{C_{k l} \in N_{r}(i, j)} \bar{D}_{i j}^{k l}+e^{\alpha \tau} \sum_{C_{k l} \in N_{r}(i, j)} \bar{E}_{i j}^{k l}\right)\right]\left|x_{i j}(t)-y_{i j}(t)\right|
\end{aligned}
$$$$
<0 \text {. }
$$

The above inequality yields $W(t) \leq W(0)$ for $t \geq 0$, which in turn implies that

$$
\begin{aligned}
\sum_{(i, j)}\left|x_{i j}(t)-y_{i j}(t)\right| e^{\alpha t} \leq & W(t) \leq W(0) \\
= & \sum_{(i, j)}\left[\left|x_{i j}(0)-y_{i j}(0)\right|+S_{i j}\left(\sum_{C_{k l} \in N_{r}(i, j)} \bar{D}_{i j}^{k l}\right.\right. \\
& \left.\left.+\sum_{C_{k l} \in N_{r}(i, j)} \bar{E}_{i j}^{k l}\right) \int_{-\tau_{k l}}^{0}\left|f_{i j}\left(x_{k l}(s)\right)-f_{i j}\left(y_{k l}(s)\right)\right| e^{\alpha\left(s+\tau_{k l}\right)} d s\right]
\end{aligned}
$$




$$
\begin{aligned}
\leq & \sum_{(i, j)}\left[\left|x_{i j}(0)-y_{i j}(0)\right|+S_{i j} L^{f}\left(\sum_{C_{k l} \in N_{r}(i, j)} \bar{D}_{i j}^{k l}\right.\right. \\
& \left.\left.+\sum_{C_{k l} \in N_{r}(i, j)} \bar{E}_{i j}^{k l}\right) \int_{-\tau}^{0}\left|x_{k l}(s)-y_{k l}(s)\right| e^{\alpha(s+\tau)} d s\right] \\
\leq & \left(1+\tau \eta(E+D) e^{\alpha \tau}\right)\|\rho(s)-\xi(s)\|,
\end{aligned}
$$

where $E=\max _{(i, j)}\left\{\max _{(k, l)} \bar{E}_{i j}^{k l}\right\}, D=\max _{(i, j)}\left\{\max _{(k, l)} \bar{D}_{i j}^{k l}\right\}$, and $\eta=\max _{(i, j)}\left\{S_{i j} L^{f}\right\}$ are constants. Further, set $\Omega=1+\tau \eta(E+D) e^{\alpha \tau}$. Then the last inequality implies that

$$
\|x(t)-y(t)\| \leq\left(1+\tau \eta(E+D) e^{\alpha \tau}\right)\|\rho(s)-\xi(s)\| e^{-\alpha t}=\Omega\|\rho(s)-\xi(s)\| e^{-\alpha t}, \quad \text { for } t \geq 0 .
$$

Therefore, the network (1) has a unique $\omega$-periodic solution which is globally exponentially stable.

Remark 4.1 Due to its practical applications in learning theory periodic solutions of FCNNs have been widely investigated by many researchers. By constructing suitable Lyapunov-Krasovskii functionals and employing the linear matrix inequality the authors in [31] considered existence and exponential stability of FCNNs. By means of M-matrix theory and differential inequality techniques Bao studied the existence and stability of a periodic solutions to BAM fuzzy Cohen-Grossberg neural networks with mixed delays. $\mathrm{Xu}$ and Li investigated global exponential stability of periodic solution for fuzzy cellular neural networks with distributed delays and variable coefficients [32]. It worth mentioning that it is the first time the existence of a periodic solutions has been considered for FSICNNs. The model of FSICNN was investigated in its general from, i.e., with time-varying coefficients and constant delays.

\section{An example}

Finally, we take into account an example with simulations of a neural system consisting of two FSICNNs. To this end, we consider the following network:

$$
\begin{aligned}
\dot{x}_{i j}(t)= & -a_{i j}(t) x_{i j}(t)-\sum_{C_{k l} \in N_{r}(i, j)} C_{i j}^{k l}(t) f_{i j}\left(x_{k l}(t)\right) x_{i j}(t)+L_{i j}(t) \\
& +\sum_{C_{k l} \in N_{r}(i, j)} B_{i j}^{k l}(t) U_{i j}(t)-\bigwedge_{C_{k l} \in N_{r}(i, j)} D_{i j}^{k l}(t) f_{i j}\left(x_{k l}\left(t-\tau_{k l}\right)\right) x_{i j}(t) \\
& -\bigvee_{C_{k l} \in N_{r}(i, j)} E_{i j}^{k l}(t) f_{i j}\left(x_{k l}\left(t-\tau_{k l}\right)\right) x_{i j}(t) \\
& +\bigwedge_{C_{k l} \in N_{r}(i, j)} T_{i j}^{k l}(t) U_{i j}(t)+\bigvee_{C_{k l} \in N_{r}(i, j)} H_{i j}^{k l}(t) U_{i j}(t),
\end{aligned}
$$

where $r=1, i, j=1,2$, and the functions $a_{i j}(t), C_{i j}(t), L_{i j}(t), B_{i j}(t), U_{i j}(t), D_{i j}(t), E_{i j}(t), T_{i j}(t)$, and $H_{i j}(t)$ are given by

$$
\left(\begin{array}{ll}
a_{11}(t) & a_{12}(t) \\
a_{21}(t) & a_{22}(t)
\end{array}\right)=\left(\begin{array}{cc}
4.36 & 1.38 \\
6 & 9.37
\end{array}\right),
$$




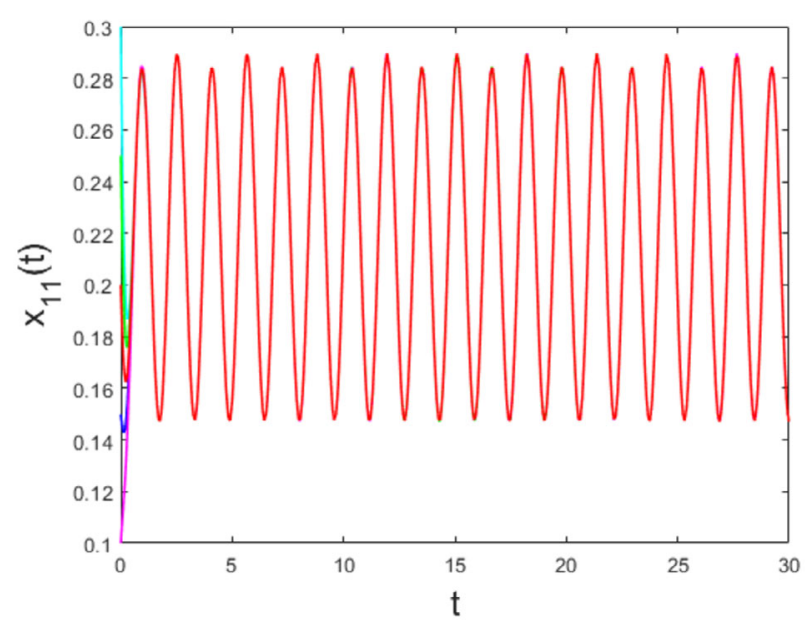

Figure 1 The state trajectory $x_{11}(t)$ of the network (11) with the initial conditions $x_{11}(t)=0.1,0.15,0.2,0.25$, and 0.3 for $t \in[-1,0]$. It is seen that the FSICNN (11) has a globally exponentially stable $\pi$-periodic solution

$$
\begin{aligned}
\left(\begin{array}{ll}
C_{11}(t) & C_{12}(t) \\
C_{21}(t) & C_{22}(t)
\end{array}\right) & =\left(\begin{array}{cc}
0.6 & 0.5 \sin ^{2}(2 t) \\
0.5 \cos ^{2}(2 t) & 0.2 \sin (2 t)
\end{array}\right) \\
\left(\begin{array}{ll}
L_{11}(t) & L_{12}(t) \\
L_{21}(t) & L_{22}(t)
\end{array}\right) & =\left(\begin{array}{cc}
\sin (2 t) & \cos (2 t) \\
0.5 \cos (2 t) & 2
\end{array}\right) \\
\left(\begin{array}{ll}
B_{11}(t) & B_{12}(t) \\
B_{21}(t) & B_{22}(t)
\end{array}\right) & =\left(\begin{array}{cc}
0.8+\cos (2 t) & 0.5-\sin (2 t) \\
0.3+\sin (2 t) & 0.1-\cos (2 t)
\end{array}\right) \\
\left(\begin{array}{ll}
U_{11}(t) & U_{12}(t) \\
U_{21}(t) & U_{22}(t)
\end{array}\right) & =\left(\begin{array}{cc}
0.3 \sin ^{2}(2 t) & 0.5 \cos (2 t) \\
0.6 \sin (2 t) & 0.3 \cos (2 t)
\end{array}\right) \\
\left(\begin{array}{ll}
D_{11}(t) & D_{12}(t) \\
D_{21}(t) & D_{22}(t)
\end{array}\right) & =\left(\begin{array}{cc}
0.8+\sin ^{2}(2 t) & 0.1 \\
0.1+0.05 \cos ^{2}(2 t) & 0.3+\cos ^{2}(2 t)
\end{array}\right) \\
\left(\begin{array}{ll}
E_{11}(t) & E_{12}(t) \\
E_{21}(t) & E_{22}(t)
\end{array}\right) & =\left(\begin{array}{cc}
0.3-\sin ^{2}(2 t) & 0.5+0.04 \cos ^{2}(2 t) \\
0.7 & 0.5 \cos ^{2}(2 t)
\end{array}\right), \\
\left(\begin{array}{ll}
T_{11}(t) & T_{12}(t) \\
T_{21}(t) & T_{22}(t)
\end{array}\right) & =\left(\begin{array}{cc}
0.09 & 5+\cos (2 t) \\
6-\sin (2 t) & 0.1+\sin ^{2}(2 t)
\end{array}\right) \\
\left(\begin{array}{ll}
H_{11}(t) & H_{12}(t) \\
H_{21}(t) & H_{22}(t)
\end{array}\right) & =\left(\begin{array}{cc}
0.2 \cos ^{2}(2 t) & 0.5+0.4 \sin ^{2}(2 t) \\
0.006-\cos ^{2}(2 t) & 1.01
\end{array}\right) .
\end{aligned}
$$

It is easy to see that the above functions are $\pi$-periodic and satisfy the condition (A1). On the other hand, one can verify that $\sum_{C_{h l} \in N_{1}(i, j)} C_{i j}^{h l}(t)=1.1+0.2 \sin (2 t), \sum_{C_{h l} \in N_{1}(i, j)} B_{i j}^{h l}(t)=$ 1.7, $\bigwedge_{C_{k l} \in N_{r}(i, j)} D_{i j}^{k l}(t)=0.1, \quad \bigvee_{C_{k l} \in N_{r}(i, j)} E_{i j}^{k l}(t)=0.7, \bigwedge_{C_{k l} \in N_{r}(i, j)} T_{i j}^{k l}(t)=0.09$, and $\bigvee_{C_{k l} \in N_{r}(i, j)} H_{i j}^{k l}(t)=1.01$.

We consider the FSICNN (11) with $f_{i j}(x)=\tanh x$ and $\tau_{11}=0.5, \tau_{12}=\pi / 5, \tau_{21}=2$, and $\tau_{22}=\pi$. One can verify that the function $\tau(\cdot)$ satisfy the condition (A1) and the nonlinear function $f(\cdot)$ satisfy the conditions (A2)-(A3). Therefore, the dynamics of the network (11) has a unique globally exponentially stable $\pi$-periodic solution according to Theorem 3.1. 


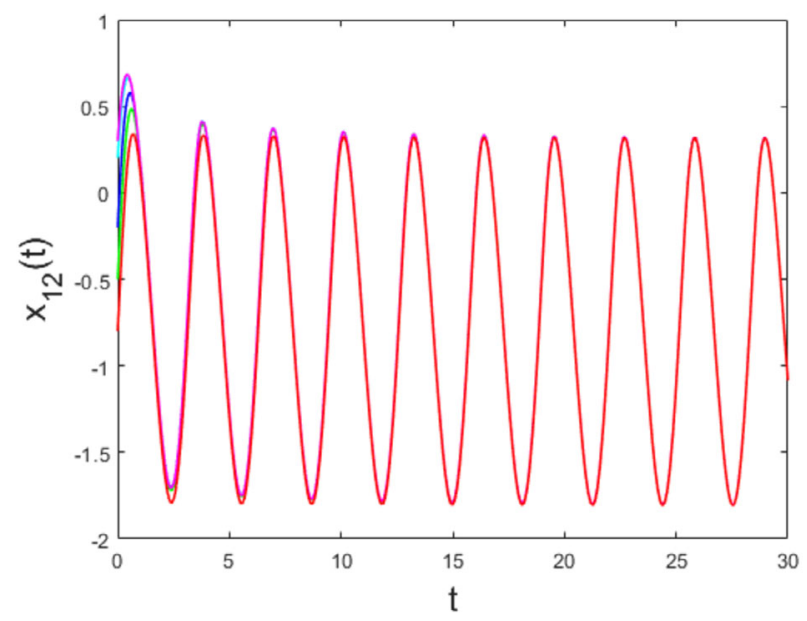

Figure 2 The state trajectory $x_{12}(t)$ of the network (11) with the initial conditions $x_{12}(t)=-0.8,-0.5,-0.1,0.3$, and 0.4 for $t \in[-1,0]$. It is seen that the FSICNN (11) has a globally exponentially stable $\pi$-periodic solution

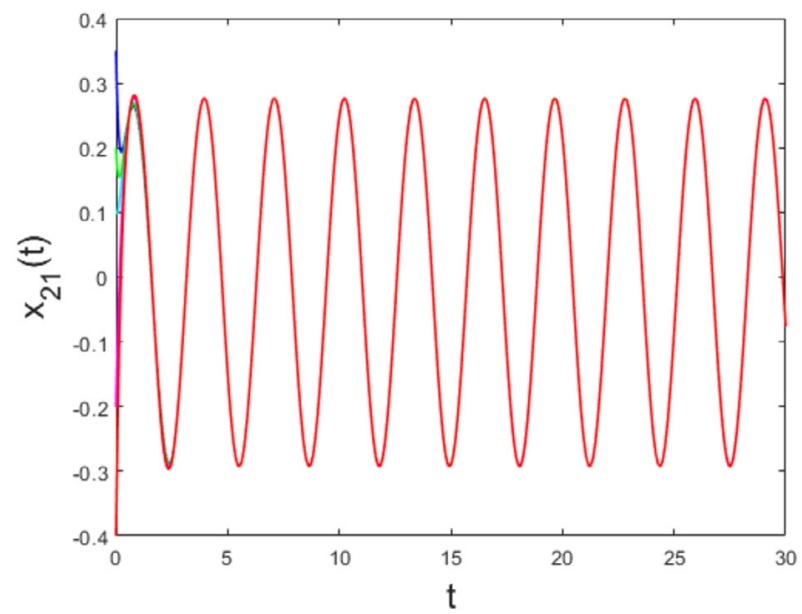

Figure 3 The state trajectory $x_{21}(t)$ of the network (11) with the initial conditions $x_{21}(t)=-0.4,-0.2,0.1,0.2$, and 0.3 for $t \in[-1,0]$. It is seen that the FSICNN (11) has a globally exponentially stable $\pi$-periodic solution

Finally, the numerical simulations in Figure 1, Figure 2, Figure 3 and Figure 4 validate our theoretical results.

\section{Conclusion}

In this paper, we established sufficient conditions for the existence of periodic solutions of FSICNNs by means of Gaines and Mawhin's coincidence degree of index zero. We considered FSICNNs with time-varying coefficients and delays. Further, by constructing Lyapunov functional we obtained sufficient condition for the exponential stability of the periodic solutions. This study can be extended further by considering FSICNNs with different type of delays such as continuously distributed delays or involving leakage term. 


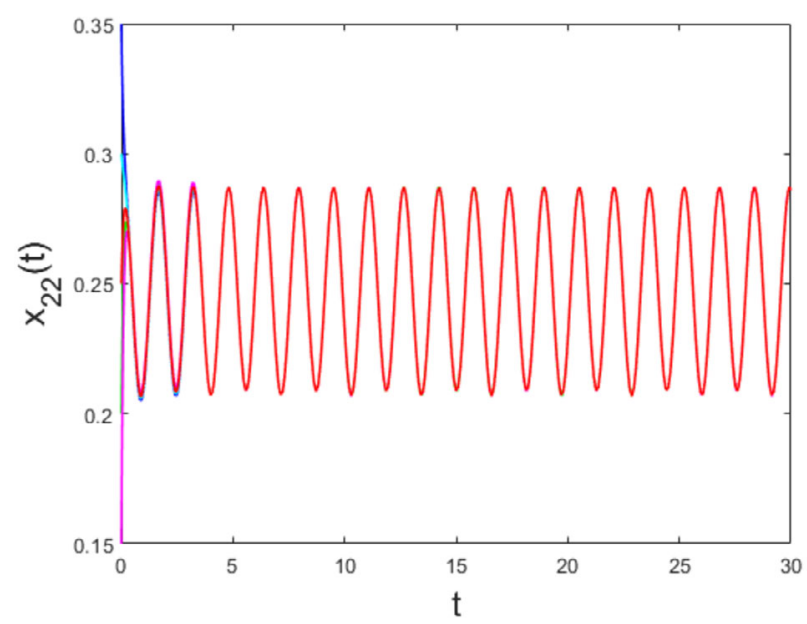

Figure 4 The state trajectory $x_{22}(t)$ of the network (11) with the initial conditions $x_{22}(t)=0.15,0.2,0.25,0.3$, and 0.35 for $t \in[-1,0]$. It is seen that the FSICNN (11) has a globally exponentially stable $\pi$-periodic solution

Funding

The second author was supported by the National Natural Science Foundation of China under Grant Nos. 61833005, 61573096, and the Jiangsu Provincial Key Laboratory of Networked Collective Intelligence under Grant BM2017002.

\section{Competing interests}

The authors declare that they have no competing interests.

Authors' contributions

All authors contributed equally to the manuscript. All authors read and approved the final version of the manuscript.

\section{Author details}

${ }^{1}$ Department of Mathematics, Nazarbayev University, Nur-Sultan, Kazakhstan. ${ }^{2}$ School of Mathematics, Southeast University, Nanjing, China.

\section{Publisher's Note}

Springer Nature remains neutral with regard to jurisdictional claims in published maps and institutional affiliations.

Received: 15 April 2019 Accepted: 28 August 2019 Published online: 05 September 2019

\section{References}

1. Wang, W., Yu, X., Luo, X., Li, L.: Stability analysis of memristive multidirectional associative memory neural networks and applications in information storage. Mod. Phys. Lett. B 32(18), Article ID 1850207 (2018), https://doi.org/10.1142/S021798491850207X

2. Zheng, M., Li, L., Peng, H., Xiao, J., Yang, Y., Zhao, H.: Finite-time stability analysis for neutral-type neural networks with hybrid time-varying delays without using Lyapunov method. Neurocomputing 238, 67-75 (2017) https://doi.org/10.1016/j.neucom.2017.01.037

3. Zhao, K., Li, Y.: Robust stability analysis of fuzzy neural network with delays. Math. Probl. Eng. 2009, Article ID 826908 (2009)

4. Zhao, K., Li, Y.: Existence and global exponential stability of equilibrium solution to reaction-diffusion recurrent neural networks on time scales. Discrete Dyn. Nat. Soc. 2010, Article ID 624619 (2010)

5. Li, Y., Zhao, K.: Robust stability of delayed reaction-diffusion recurrent neural networks with Dirichlet boundary conditions on time scales. Neurocomputing 74(10), 1632-1637 (2011). https://doi.org/10.1016/..neucom.2011.01.006

6. Li, Y., Zhao, K., Ye, Y.: Stability of reaction-diffusion recurrent neural networks with distributed delays and Neumann boundary conditions on time scales. Neural Process. Lett. 36, 217 (2012). https://doi.org/10.1007/s11063-012-9232-2

7. Zhao, K., Wang, L., Liu, J.: Global robust attractive and invariant sets of fuzzy neural networks with delays and impulses. J. Appl. Math. 2013, Article ID 935491 (2013)

8. Zhao, K.: Global robust exponential synchronization of BAM recurrent FNNs with infinite distributed delays and diffusion terms on time scales. Adv. Differ. Equ. 2014, 317 (2014). https://doi.org/10.1186/1687-1847-2014-317

9. Cao, J.: Global asymptotic stability of neural networks with transmission delays. Int. J. Inf. Syst. Sci. 31(10), 1313-1316 (2000). https://doi.org/10.1080/00207720050165807

10. Yang, T., Yang, L.B., Wu, C., Chua, L.O.: Fuzzy cellular neural networks: theory. In: 1996 Fourth IEEE International Workshop on Cellular Neural Networks and Their Applications Proceedings (CNNA-96), pp. 181-186 (1996). https://doi.org/10.1109/CNNA.1996.566545 
11. Yang, T., Yang, L.B., Wu, C.W., Chua, L.O.: Fuzzy cellular neural networks: applications. In: 1996 Fourth IEEE International Workshop on Cellular Neural Networks and Their Applications Proceedings (CNNA-96), pp. 225-230 (1996). https://doi.org/10.1109/CNNA.1996.566560

12. Liu, Y., Tang, W.: Exponential stability of fuzzy cellular neural networks with constant and time-varying delays. Phys. Lett. A 323(3), 224-233 (2004). https://doi.org/10.1016/j.physleta.2004.01.064

13. Song, Q., Cao, J.: Impulsive effects on stability of fuzzy Cohen-Grossberg neural networks with time-varying delays. IEEE Trans. Syst. Man Cybern., Part B, Cybern. 37(3), 733-741 (2007). https://doi.org/10.1109/TSMCB.2006.887951

14. Huang, T.: Exponential stability of fuzzy cellular neural networks with distributed delay. Phys. Lett. A 351(1), 48-52 (2006). https://doi.org/10.1016/j.physleta.2005.10.060

15. Xu, C., Li, P.: Exponential stability for fuzzy BAM cellular neural networks with distributed leakage delays and impulses. Adv. Differ. Equ. 2016(1), 276 (2016). https://doi.org/10.1186/s13662-016-0978-0

16. Syed Ali, M., Balasubramaniam, P.: Global asymptotic stability of stochastic fuzzy cellular neural networks with multiple discrete and distributed time-varying delays. Commun. Nonlinear Sci. Numer. Simul. 16(7), 2907-2916 (2011). https://doi.org/10.1016/j.cnsns.2010.10.011

17. Liu, Z., Zhang, H., Wang, Z.: Novel stability criterions of a new fuzzy cellular neural networks with time-varying delays. Neurocomputing 72(4), 1056-1064 (2009) Brain Inspired Cognitive Systems (BICS 2006) / Interplay Between Natural and Artifficial Computation (IWINAC 2007). https://doi.org/10.1016/j.neucom.2008.04.001

18. Balasubramaniam, P., Rakkiyappan, R., Sathy, R.: Delay dependent stability results for fuzzy BAM neural networks with Markovian jumping parameters. Expert Syst. Appl. 38(1), 121-130 (2011). https://doi.org/10.1016/j.eswa.2010.06.025

19. Tan, M.: Global asymptotic stability of fuzzy cellular neural networks with unbounded distributed delays. Neural Process. Lett. 31(2), 147-157 (2010). https://doi.org/10.1007/s11063-010-9130-4

20. Balasubramaniam, P., Kalpana, M., Rakkiyappan, R.: State estimation for fuzzy cellular neural networks with time delay in the leakage term, discrete and unbounded distributed delays. Comput. Math. Appl. 62(10), 3959-3972 (2011). https://doi.org/10.1016/j.camwa.2011.09.048

21. Chen, L., Wu, L., Pan, D.: Mean square exponential stability of impulsive stochastic fuzzy cellular neural networks with distributed delays. Expert Syst. Appl. 38(5), 6294-6299 (2011). https://doi.org/10.1016/j.eswa.2010.11.070

22. Wang, J., Lu, J.G.: Global exponential stability of fuzzy cellular neural networks with delays and reaction-diffusion terms. Chaos Solitons Fractals 38(3), 878-885 (2008). https://doi.org/10.1016/j.chaos.2007.01.032

23. Park, M.J., Kwon, O.M., Park, Ju H., Lee, S.M.: Simplified stability criteria for fuzzy Markovian jumping Hopfield neural networks of neutral type with interval time-varying delays. Expert Syst. Appl. 39(5), 5625-5633 (2012). https://doi.org/10.1016/j.eswa.2011.11.055

24. Ding, W., Han, M.: Synchronization of delayed fuzzy cellular neural networks based on adaptive control. Phys. Lett. A 372(26), 4674-4681 (2008). https://doi.org/10.1016/j.physleta.2008.04.053

25. Abdurahman, A., Jiang, H., Teng, Z.: Finite-time synchronization for fuzzy cellular neural networks with time-varying delays. Fuzzy Sets Syst. 297, 96-111 (2016) Themed Section: Fuzzy Systems. https://doi.org/10.1016/j.fss.2015.07.009

26. Wang, W.: Finite-time synchronization for a class of fuzzy cellular neural networks with time-varying coefficients and proportional delays. Fuzzy Sets Syst. 338, 40-49 (2018) Theme: Fuzzy Systems. https://doi.org/10.1016/j.fss.2017.04.005

27. Gan, Q., Xu, R., Yang, P.: Exponential synchronization of stochastic fuzzy cellular neural networks with time delay in the leakage term and reaction-diffusion. Commun. Nonlinear Sci. Numer. Simul. 17(4), 1862-1870 (2012). https://doi.org/10.1016/j.cnsns.2011.08.029

28. Yu, F., Jiang, H.: Global exponential synchronization of fuzzy cellular neural networks with delays and reaction-diffusion terms. Neurocomputing 74(4), 509-515 (2011). https://doi.org/10.1016/j.neucom.2010.08.017

29. Yu, J., Hu, C., Jiang, H., Teng, Z.: Exponential lag synchronization for delayed fuzzy cellular neural networks via periodically intermittent control. Math. Comput. Simul. 82(5), 895-908 (2012). https://doi.org/10.1016/j.matcom.2011.11.006

30. Yan, P., Lv, T.: Exponential synchronization of fuzzy cellular neural networks with mixed delays and general boundary conditions. Commun. Nonlinear Sci. Numer. Simul. 17(2), 1003-1011 (2012). https://doi.org/10.1016/j.cnsns.2011.07.013

31. Yuan, K., Cao, J., Deng, J.: Exponential stability and periodic solutions of fuzzy cellular neural networks with time-varying delays. Neurocomputing 69(13), 1619-1627 (2006) Blind Source Separation and Independent Component Analysis. https://doi.org/10.1016/j.neucom.2005.05.011

32. Xu, C., Li, P.: Global exponential stability of periodic solution for fuzzy cellular neural networks with distributed delays and variable coefficients. J. Intell. Fuzzy Syst. 32(3), 2603-2615 (2017)

33. Huang, Z:: Almost periodic solutions for fuzzy cellular neural networks with multi-proportional delays. Int. J. Mach. Learn. Cybern. 8(4), 1323-1331 (2017). https://doi.org/10.1007/s13042-016-0507-1

34. Bao, H.: Existence and exponential stability of periodic solution for BAM fuzzy Cohen-Grossberg neural networks with mixed delays. Neural Process. Lett. 43(3), 871-885 (2016). https://doi.org/10.1007/s11063-015-9455-0

35. Bouzerdoum, A., Pinter, R.B.: Shunting inhibitory cellular neural networks: derivation and stability analysis. IEEE Trans. Circuits Syst. I, Fundam. Theory Appl. 40(3), 215-221 (1993). https://doi.org/10.1109/81.222804

36. Cheung, H.N., Bouzerdoum, A., Newland, W.: Properties of shunting inhibitory cellular neural networks for colour image enhancement. In: 6th International Conference on Neural Information Processing, 1999, pp. 89-94 (1999)

37. Arulampalam, G., Bouzerdoum, A.: Application of shunting inhibitory artificial neural networks to medical diagnosis In: The Seventh Australian and New Zealand Intelligent Information Systems Conference, 2001, pp. 89-94 (2001). https://doi.org/10.1109/ANZIIS.2001.974056

38. Chen, A., Cao, J.: Almost periodic solution of shunting inhibitory CNNs with delays. Phys. Lett. A 298(2), 161-170 (2002). https://doi.org/10.1016/S0375-9601(02)00469-3

39. Chen, L., Zhao, H.: Global stability of almost periodic solution of shunting inhibitory cellular neural networks with variable coefficients. Chaos Solitons Fractals 35(2), 351-357 (2008). https://doi.org/10.1016/j.chaos.2006.05.057

40. Ding, H.-S., Liang, J., Xiao, T.-J.: Existence of almost periodic solutions for SICNNs with time-varying delays. Phys. Lett. A 372(33), 5411-5416 (2008). https://doi.org/10.1016/j.physleta.2008.06.042

41. Li, Y., Liu, C., Zhu, L.: Global exponential stability of periodic solution for shunting inhibitory CNNs with delays. Phys. Lett. A 337(1), 46-54 (2005). https://doi.org/10.1016/j.physleta.2005.01.008 
42. Zhou, Q., Xiao, B., Yu, Y., Peng, L.: Existence and exponential stability of almost periodic solutions for shunting inhibitory cellular neural networks with continuously distributed delays. Chaos Solitons Fractals 34(3), 860-866 (2007). https://doi.org/10.1016/j.chaos.2006.03.092

43. Akhmet, M.U., Fen, M.O.: Shunting inhibitory cellular neural networks with chaotic external inputs. Chaos, Interdiscip. J. Nonlinear Sci. 23(2), 023112 (2013). https://doi.org/10.1063/1.4805022

44. Akhmet, M., Fen, M.O., Kivilcim, A.: Li-Yorke chaos generation by SICNNs with chaotic/almost periodic postsynaptic currents. Neurocomputing 173, 580-594 (2016). https://doi.org/10.1016/j.neucom.2015.08.001

45. Gaines, R., Mawhin, J.L.: Coincidence Degree and Nonlinear Differential Equations. Springer, New York (1977)

46. Yang, T., Yang, L.B.: The global stability of fuzzy cellular neural network. IEEE Trans. Circuits Syst. I, Fundam. Theory Appl. 43(10), 880-883 (1996). https://doi.org/10.1109/81.538999

Submit your manuscript to a SpringerOpen ${ }^{\circ}$ journal and benefit from:

- Convenient online submission

- Rigorous peer review

- Open access: articles freely available online

- High visibility within the field

- Retaining the copyright to your article

Submit your next manuscript at $\gg$ springeropen.com 